\title{
Longitudinal Association between Selenium Levels and Hypertension in a Rural Elderly Chinese Cohort
}

\author{
Liqin $\mathrm{Su}^{1}$, Yinlong Jin, ${ }^{1}$ Frederick W. Unverzagt, ${ }^{2}$ Chaoke Liang, ${ }^{1}$ Yibin Cheng, ${ }^{1}$ Ann M. Hake, ${ }^{3}$ Dulanji \\ Kuruppu $^{3}$, Feng Ma, ${ }^{1}$ Jingyi Liu, ${ }^{1}$ Chen Chen, ${ }^{1}$ Jianchao Bian, ${ }^{4}$ Ping Li, ${ }^{5}$ Sujuan Gao ${ }^{6}$
}

\footnotetext{
${ }^{1}$ Institute for Environmental Health and Related Product Safety, Chinese Center for Disease Control and Prevention, Beijing, China

${ }^{2}$ Department of Psychiatry, Indiana University School of Medicine, Indianapolis, IN, United States

${ }^{3}$ Department of Neurology, Indiana University School of Medicine, Indianapolis, IN, United States, ${ }^{4}$ Shandong Institute for Prevention and Treatment of Endemic Disease in China, Jinan, China

${ }^{5}$ Sichuan Provincial Center for Disease Control and Prevention in China, Chengdu, China

${ }^{6}$ Department of Biostatistics, Indiana University School of Medicine, Indianapolis, IN, United States
}

\section{Corresponding authors:}

Dr. Sujuan Gao, Department of Biostatistics, Indiana University School of Medicine, 410 West $10^{\text {th }}$ Street, \#3000, Indianapolis, IN 46202-2872, Phone: U.S. (317) 274-0820 Fax: (317)274-2678, Email: sgao@iu.edu.

\section{Financial support:}

This work was supported by the National Institute of Health (R01 AG019181).

Total word count: 4496

Tables: 4

Figures: 2

This is the author's manuscript of the article published in final edited form as: Su, L., Jin, Y., Unverzagt, F. W., Liang, C., Cheng, Y., Hake, A. M., .. Gao, S. (2016). Longitudinal association between selenium levels. The Journal of Nutrition, Health \& Aging, 1-6. http://doi.org/10.1007/s12603-016-0700-7 


\begin{abstract}
Objectives: Results from previous studies have been inconsistent on the association between selenium and hypertension, and very few studies on this subject have focused on the elderly population. The purpose of this study is to examine the relationship between selenium level and hypertension in a rural elderly Chinese cohort.
\end{abstract}

Design: A longitudinal study was implemented and data were analyzed using logistic regression models and Cox proportional hazards regression model adjusting for potential confounders. The association between selenium level and prevalent hypertension at baseline and between selenium and incident hypertension were examined.

Setting: Community-based setting in four rural areas in China.

Subjects: A total of 2000 elderly aged 65 years and over (mean $71.9 \pm 5.6$ years) participated in this study.

Measurements: Nail selenium levels were measured in all subjects at baseline. Blood pressure measures and self-reported hypertension history were collected at baseline, 2.5 years and 7 years later. Hypertension was defined as systolic blood pressure $140 \mathrm{mmHg}$ or higher, diastolic blood pressure $90 \mathrm{mmHg}$ or higher, or reported use of anti-hypertensive medication.

Results: The rate of baseline hypertension was $63.50 \%$ in this cohort and the mean nail selenium level is $0.413 \pm 0.183 \mu \mathrm{g} / \mathrm{g}$. Multi-covariate adjusted cross-sectional analyses indicated that higher selenium level was associated with higher blood pressure measures at baseline and higher rates of hypertension. For the 635 participants with normal blood pressure at baseline, 360 had developed hypertension during follow-up. The incidence rate for hypertension was $45.83 \%$, $52.27,62.50 \%, 70.48 \%$, and $62.79 \%$ from the first selenium quintile to the fifth quintile respectively. Comparing to the lowest quintile group, the hazard ratio were $1.41(95 \% \mathrm{CI}: 1.03$ 
to1.94), 1.93 (95\%CI: 1.40 to 2.67$), 2.35$ (95\%CI: 1.69 to 3.26$)$ and 1.94 (95\%CI: 1.36 to 22.77$)$ for the second selenium quintile to the fifth quintile respectively.

Conclusions: Our findings suggest that high selenium may play a harmful role in the development of hypertension. Future studies are needed to confirm our findings and to elucidate a plausible biological mechanism.

Keywords: Selenium; Hypertension; Blood pressure; Elderly population; Environmental exposure 


\section{Introduction}

Hypertension affects nearly one billion people worldwide, many of them over the age of 65, and contributes to the global burden of disease and mortality [1]. Studies have shown that oxidative stress is one of the fundamental mechanisms underlying hypertension [2]. Antioxidants inhibit oxidation reactions, thereby reducing the number of free radicals produced and the amount of damage they can cause. Selenium, an essential trace element with antioxidant properties [3], was hypothesized to have a protective effect on hypertension [4].

A link between selenium and hypertension was provided in selenium's role in Keshan disease, a disorder that occurred in regions of China where selenium was severely deficient in the soil and diet, the symptoms of Keshan disease, including hypertension, can be relieved by administering selenium supplements [5]. There are reports from observational studies of higher selenium levels associated with lower blood pressure [6-8]. However, other studies have shown that in populations where selenium levels exceed the recommended daily intake, higher selenium levels were found to be associated with higher blood pressure and higher rates of hypertension [9-11]. It should be noted that few of published studies are longitudinal in design. In addition to the inconsistency in findings on the relationship between selenium and blood pressure, very few studies on this subject have focused on the elderly population despite the considerable proportion of the elderly suffering from hypertension. The objective of this study was to examine the relationship between selenium level and hypertension in an elderly Chinese population.

\section{Materials and methods}

\section{Study design and participants}

Participants were recruited for the Selenium and Cognitive Decline study, a longitudinal epidemiologic project funded by the National Institute of Health examining the long-term impact 
of selenium on cognitive decline in rural elderly Chinese. Participants were enrolled between 2003 and 2005, and underwent two follow-up evaluations at 2.5 years and 7 years after baseline. Two counties from Sichuan Province in southwestern China, and two counties from Shandong Province in eastern China were selected for the project. These study sites were selected for the following reasons: (1). They were rural counties; (2). The two counties within a province differed in selenium levels but were similar in demographics and other trace element measures; and (3). The four counties were also required to have sufficient population so that the local elderly population was large enough to provide a sample of 500 elderly subjects. Sites with known endemic diseases (including Keshan disease, Kaschin-Beck disease, goiter and Cretinism, and fluorosis) were excluded from consideration. Details on site selection process were described previously [12].

Residents aged 65 years or older from these four counties were invited to participate in the study. The only exclusion criterion is severe hearing loss which makes it impossible to conduct cognitive assessment. For each county included in the study, interviews were conducted from village to village, and completed when 500 elderly participants were enrolled. The total sample size was 2000 at baseline, 95 participants were lost during the 2.5 year follow-up because of death. The study was approved by Indiana University Institutional Review Board and the Institute for Environmental Health and Related Safety, Chinese Center for Disease Control and Prevention.

\section{Hypertension and Blood Pressure Measurements}

During the interview, participants were asked whether they were ever told by a doctor or a health care professional that they had hypertension or high blood pressure defined as systolic blood pressure $\geqslant 140 \mathrm{mmHg}$, or diastolic blood pressure $\geqslant 90 \mathrm{mmHg}$. A follow-up question of 
whether they were taking any anti-hypertensive medications was asked if they answered "yes" to the hypertension question.

A physical examination was also conducted during the interview. Two consecutive measurements of systolic and diastolic blood pressure were taken by trained interviewers using a mercury sphygmomanometer while the participant was seated following the protocol outlined in the Hypertension Detection and Follow-up Program and used in other studies [13]. The average of the two blood pressure measurements was used in all analyses. The hypertension and blood pressure measures were collected at baseline, 2.5 and 7 years after baseline. In this analysis, hypertension was defined as systolic blood pressure $140 \mathrm{mmHg}$ or higher, diastolic blood pressure $90 \mathrm{mmHg}$ or higher, or reported use of anti-hypertensive medication.

\section{Selenium Measures}

Nail samples were collected at the time of interview and stored in clean plastic bags during the baseline survey. Fluorometric determination of trace amount of selenium with 2, 3diaminonaphthalene was used to determine trace amounts of selenium in nails. The details on laboratory procedures and quality control measures were described elsewhere [12].

\section{Covariates}

Other information collected during the survey included age, gender, education (whether the participant had attended school), alcohol consumption and smoking history. Participants' height and weight were also measured during the interview. Body Mass Index (BMI, defined as body weight in kilograms divided by height in meters squared) was derived from height and weight measurements. The International Physical Activity Questionnaire (IPAQ) was added during the 2.5-year and 7-year follow-up survey. Physical activity was classified into the three categories of 
low, moderate and high according to the Guidelines for Data Processing and Analysis of the IPAQ [14].

\section{Statistical analysis}

To capture potential non-linear relationships between selenium levels and blood pressure measures, the study population was divided into quintiles according to nail selenium levels. In addition to selenium levels, we considered the following variables potential confounding factors possibly related to both blood pressure and selenium levels: age, gender, education, BMI, smoking, alcohol consumption and IPAQ.

Analysis of variance (ANOVA) was used to compare the means of continuous variables and chisquare test was used to compare proportions of categorical variables across selenium quintile groups. Analysis of covariance (ANCOVA) models were used to determine the association between selenium level and baseline blood pressure adjusting for other covariates. Logistic regression models were used to determine the association between selenium levels and baseline hypertension adjusting for the aforementioned confounders.

Participants who were normotensive at baseline were included in the longitudinal analysis. The incidence rates of hypertension were compared among the five selenium quintile groups using chi-square test. Time to incident hypertension during the 7-year follow-up was used as the outcome variable in Cox proportional hazards regression models to examine the effect of baseline selenium level on the incidence of hypertension. Participants who remained normotensive were censored at the last evaluation.

All analyses were performed using SAS9.1 for Windows (SAS Institute Inc., Cary, North Carolina, USA). $P<0.05$ was considered statistically significant. 


\section{Results}

For the baseline sample of 2000 participants, mean age was $71.9 \pm 5.6$ and mean nail selenium level is $0.413 \pm 0.183 \mu \mathrm{g} / \mathrm{g}$. Among the five nail selenium quintile groups significant differences were seen on gender, education, alcohol consumption, smoking, BMI, systolic blood pressure, diastolic blood pressure and hypertension rate, while no difference in age was observed, as shown in Table 1 . The rate of hypertension was $63.50 \%$ for the total population at baseline. Hypertension rates at baseline ranged from $43.78 \%, 62.22 \%, 66.33 \%, 69.52 \%$, to $75.81 \%$ with the increasing order of selenium level in the selenium quintile groups respectively (Figure 1).

Univariate analysis indicated that the males, smokers and alcohol consumers had significantly lower selenium level compared with the females, non-smokers and non-alcohol consumers, respectively and that selenium was positively associated with BMI $(P<0.05$ for all comparisons. Results not shown). Selenium level in participants with hypertension was significantly higher than in the normotensive, with mean selenium levels $0.442 \pm 0.181 \mu \mathrm{g} / \mathrm{g}$ and $0.364 \pm 0.175 \mu \mathrm{g} / \mathrm{g}$ in the hypertensive and normotensive participants, respectively.

In Table 2, we present results on the association between selenium quintiles and baseline hypertension or blood pressure adjusting for various covariates. Parameter estimates from the ANCOVA models are the estimated differences in mean blood pressure measures between each selenium quintile group and the reference group while adjusting for other covariates. Comparing to participants in the lowest selenium quintile group, those in the four other quintile groups had significantly higher systolic blood pressure and diastolic blood pressure. Higher baseline selenium is also significantly associated with hypertension with odds ratios of $2.17(95 \% \mathrm{CI}: 1.63$ to 2.90$), 2.46(95 \% \mathrm{CI}$ : 1.83 to 3.23$), 2.72(95 \% \mathrm{CI}: 2.00$ to 3.70$), 3.55$ (95\%CI: 2.59 to 4.87$)$ from the second selenium quintile to the fifth quintile, respectively. There are no differences on 
hypertension rates among the four top quintile groups since the confidence intervals of the odds ratio estimates overlap.

In the 730 participants with normal blood pressure at baseline, 635 participated in the follow-up evaluations (95 had died), 360 had developed incident hypertension. Baseline population characteristics did not differ between those with incident hypertension and the normotensive with respect to age, gender, education, alcohol consumption, smoking and diastolic blood pressure (Table 3). However, selenium level, BMI and systolic blood pressure were significantly higher in those with incident hypertension than in the normotensive.

Crude incidence rates for hypertension were $45.83 \%, 52.27,62.50 \%, 70.48 \%, 62.79 \%$ from the first quintile to the fifth selenium quintile groups, respectively (Figure 2). In Table 4, we present results of the Cox model that baseline selenium level was significantly associated with the incidence of hypertension adjusting for other covariates. Comparing to the lowest selenium quintile group, hazard ratios for incident hypertension were 1.41 (95\%CI: 1.03 to1.94), 1.93 (95\%CI: 1.40 to 2.67 ), 2.35 (95\%CI: 1.69 to 3.26 ) and 1.94 (95\%CI: 1.36 to 22.77 ) for the second selenium quintile to the fifth selenium quintile groups, respectively.

\section{Discussion}

In this study, we examined the associations between selenium level and hypertension in a 7-year follow up of elderly Chinese population in the Selenium and Cognitive Decline study. We found that participants with higher selenium levels had higher blood pressure and hypertension rate at baseline, and participants with higher baseline selenium levels also had greater risk of developing hypertension.

There were four published longitudinal studies focusing on the association between selenium and blood pressure or hypertension [8, 15-17]. In a prospective study in Belgium [8], a significant 
protective effect of selenium on incident hypertension was observed in young adult males, but no significant relationship was observed in females. The other three studies reported non-significant associations between selenium and hypertension [15-17]. A previous systematic literature review published in 2014 found no conclusive evidence supporting an association between selenium levels and hypertension [18]. Our findings are consistent with results from several crosssectional studies and case-control studies. In the US National Health and Nutrition Examination Survey data (2003-2004), high serum selenium concentrations were associated with higher prevalence of hypertension [10]. Another cross-sectional study in 3387 males aged 53-74 years also found an association between higher selenium levels and higher hypertension rates [11]. Similar result was also reported in a case-control study in France [9]. However, there were studies reporting non-significant association [19-21] while others supported an inverse association between selenium levels and hypertension $[6,22,23]$. In a cross-sectional study on 722 males with mean age 54 years old in eastern Finland higher selenium level was found to be associated with lower hypertension rate [6]. Two case-control studies reported lower selenium in hypertensive patients than in normal controls $[22,23]$. In addition to observational studies, randomized controlled trials with selenium as one of the intervention agents also reported protective effect on hypertension [24, 25].

The inconsistent results from previous studies may be partially due to the difference in selenium exposure levels among the different study populations. In most previous studies, selenium's harmful effect on hypertension was more likely to be seen in selenium-replete populations and protective effect seen in selenium-deplete populations [18]. Moreover, the definition of hypertension varied among different countries, such as Belgium [8], Denmark [11], Finland [26 ], and the United States [17]. Other factors contributing to the inconsistent results include 
differences in study design, adjustment for confounding factors and the difference in the age range covered by a study. While the biological mechanism underlying a positive association between selenium and blood pressure measure is not yet fully understood, selenium's effect on oxidative stress has been investigated in animal studies. As a key component of many antioxidant enzymes, such as glutathione peroxidase (GPx) selenium plays an important role in oxidative stress [27], which is recognized as one of the fundamental mechanisms underlying hypertension [2]. An experimental study had observed that selenium at low doses can provide significant protection of the human coronary artery endothelium against damage by oxidative stress [28]. In an animal model, dietary supplementation with selenium was associated with lower levels of cardiac oxidative damage and increased antioxidant expression, as well as a reduction in disease severity and mortality in spontaneously hypertensive rats [29]. Although experimental results mentioned above support the hypothesis that selenium deficiency is associated with oxidative stress, nonlinear dose-response relationship between selenium exposure and oxidative stress biomarkers was observed in a recent study suggesting that high selenium levels increase oxidative stress [30]. Our study, unfortunately, did not have measures of other oxidative stress markers and will not be able to examine the role of oxidative stress in the association between selenium and hypertension. More future studies on the relationship among selenium level, oxidative stress and hypertension are needed.

Thus far, most studies examining the relationship between selenium and hypertension have either included subjects over a very broad age range or have focused on middle-aged adults or children. Our study focused exclusively on the elderly population where hypertension is much more prevalent. Enrolling participants from rural areas also ensured that the majority of the 
participants in our study had lived in the same village their entire lives and they were not taking dietary supplements, thus providing a stable, long-term measure of selenium exposure.

It is important to note that nail selenium represents a more stable measure of long-term exposure to selenium [31] and selenium level in nails does not fluctuate greatly with daily selenium intake in the diet [32]. In a previous study in the same cohort [12], we have confirmed that selenium levels in nail samples were significantly correlated with selenium levels measured in blood and dietary intake derived from food frequency questionnaire. Although we are unable to compare selenium levels in our study directly with selenium levels measured in blood or hair samples in other studies, the selenium level in our cohort was similar to the Finland study of age 55-69 year old sample [32], and lower than toenail selenium levels in the American [11,33] and the Northern Italy samples [34].

\section{Strengths and limitations}

Our study has a number of strengths. The first is the prospective study design and the relatively large sample size ensuring adequate statistical power. The second is the relatively low selenium level in the study population without selenium supplementation, providing an opportunity to explore the associations between selenium exposure and hypertension. The third is the selenium measurements in nail samples, which provided a relatively long-term measure of exposure compared with selenium measured in blood or urine samples.

There are also several limitations in this study. The first is that the cohort included participants older than 65 years of age. Therefore, it is not known whether the observed association between selenium and hypertension holds in populations of younger subjects. Another limitation was that glutathione peroxidase (GPx) activity was not measured. Measuring GPx levels may enable us

to compare enzyme activity to selenium levels and gain a better understanding of the association 
between selenium and hypertension. Finally, the current study did not consider genetic factors or other environmental factors that may influence or modify the association between selenium level and the risk of hypertension.

Conclusions: Our results, both cross-sectional and longitudinal, suggest that higher selenium may be associated with the risk of hypertension. Future studies in other populations of similar or different age composition are needed to confirm our findings. In addition, studies that incorporate more biomarkers are needed to elucidate the biological mechanism underlying the association between selenium and hypertension.

\section{Acknowledgement}

The authors express thanks to all the involved staff from the Chinese local cooperative hospitals and Centers for Disease Control and Prevention in Shandong and Sichuan province for their help in the field work.

\section{Conflict of interest}

The authors declare that there are no conflicts of interests.

\section{Ethical standards}

The authors declare that all the experiments of this study complied with the current laws of China in which they were performed. 


\section{References}

1. Poulter NR, Prabhakaran D, Caulfield M. Hypertension. Lancet. 2015 Mar 27. pii: S01406736(14) 61468-1469. doi: 10.1016/S0140-6736(14)61468-1469.

2. González J, Valls N, Brito R, Rodrigo R. Essential hypertension and oxidative stress: New insights. World J Cardiol. 2014; 6(6):353-366.

3. Rayman MP. The importance of selenium to human health. Lancet. 2000; 356(9225):233241.

4. Navas-Acien A, Bleys J, Guallar E. Selenium intake and cardiovascular risk: what is new? Curr Opin Lipidol. 2008; 19(1): 43-49.

5. Boosalis MG. The role of selenium in chronic disease. Nutr Clin Pract. 2008; 23(2):152-160.

6. Salonen JT, Salonen R, Ihanainen M, Parviainen M, Seppänen R, Kantola M, et al. Blood pressure, dietary fats, and antioxidants. Am J Clin Nutr. 1988; 48(5):1226-1232.

7. Deucher GP. Antioxidant therapy in the aging process. EXS. 1992;62:428-437.

8. Nawrot TS, Staessen JA, Roels HA, Den Hond E, Thijs L, Fagard RH, et al. Blood pressure and blood selenium: a cross-sectional and longitudinal population study. Eur Heart J. 2007; 28(5):628-633.

9. Coudray C, Roussel AM, Mainard F, Arnaud J, Favier A. Lipid peroxidation level and antioxidant micronutrient status in a pre-aging population; correlation with chronic disease prevalence in a French epidemiological study (Nantes, France). J Am Coll Nutr. 1997; 16(6):584-591.

10. Laclaustra M, Navas-Acien A, Stranges S, Ordovas JM, Guallar E. Serum selenium concentrations and hypertension in the US Population. Circ Cardiovasc Qual Outcomes. 2009; 2(4):369-376. 
11. Suadicani P, Hein HO, Gyntelberg F. Serum selenium concentration and risk of ischaemic heart disease in a prospective cohort study of 3000 males. Atherosclerosis. 1992; 96(1):3342.

12. Gao S, Jin Y, Hall KS, Liang C, Unverzagt FW, Ji R, et al. Selenium level and cognitive function in rural elderly Chinese. Am J Epidemiol. 2007; 165(8):955-965.

13. Hebert LE, Scherr PA, Bennett DA, Bienias JL, Wilson RS, Morris MC, et al. Blood pressure and late-life cognitive function change: a biracial longitudinal population study. Neurology. 2004; 62(11):2021-2024.

14. IPAQ group. Guidelines for data processing and analysis of the International Physical Activity Questionnaire (IPAQ). 2005. Available: http://www.ipaq.ki.se/scoring.html.

15. Arnaud J, Akbaraly TN, Hininger I, Roussel AM, Berr C. Factors associated with longitudinal plasma selenium decline in the elderly: the EVA study. J Nutr Biochem. 2007; 18(7): 482-487.

16. Stranges S, Galletti F, Farinaro E, D'Elia L, Russo O, Iacone R,et al. Associations of selenium status with cardiometabolic risk factors: An 8-year follow-up analysis of the Olivetti Heart Study. Atherosclerosis. 2011; 217(1):274-278.

17. Xun P, Hou N, Daviglus M, Liu K, Morris JS, Shikany JM, et al. Fish oil, selenium and mercury in relation to incidence of hypertension: a 20-year follow-up study. J Intern Med. 2011; 270(2):175-186.

18. Kuruppu D, Hendrie HC, Yang L,Gao S. Selenium levels and hypertension: a systematic review of the literature. Public Health Nutr. 2014; 17(6):1342-1352. 
19. Parizadeh SM, Moohebati M, Ghafoori F, Ghayour-Mobarhan M, Kazemi-Bajestani SM, Tavallaie S, et al. Serum selenium and glutathione peroxidase concentrations in Iranian patients with angiography-defined coronary artery disease. Angiology. 2009; 60(2):186-191.

20. Pavao ML, Figueiredo T, Santos V et al. Whole blood glutathione peroxidase and erythrocyte superoxide dismutase activities, serum trace elements $(\mathrm{Se}, \mathrm{Cu}, \mathrm{Zn})$ and cardiovascular risk factors in subjects from the city of Ponta Delgada, Island of San Miguel, The Azores Archipelago, Portugal. Biomarkers. 2006; 11: 460-471.

21. Pemberton PW, Ahmad Y, Bodill H et al. Biomarkers of oxidant stress, insulin sensitivity and endothelial activation in rheumatoid arthritis: a cross-sectional study of their association with accelerated atherosclerosis. BMC Res Notes. 2009; 2: 83.

22. Li N, Gao Z, Luo D, Tang X, Chen D, Hu Y. Selenium level in the environment and the population of Zhoukoudian area, Beijing, China. Sci Total Environ. 2007; 381, 105-111.

23. Mihailović MB, Avramović DM, Jovanović IB, Pesut OJ, Matić DP, Stojanov VJ. Blood and plasma selenium levels and GSH-Px activities in patients with arterial hypertension and chronic heart disease. J Environ Pathol Toxicol Oncol. 1998; 17(3-4):285-259.

24. Shargorodsky M, Debby O, Matas Z et al. Effect of longterm treatment with antioxidants (vitamin C, vitamin E, coenzyme Q10 and selenium) on arterial compliance, humoral factors and inflammatory markers in patients with multiple cardiovascular risk factors. Nutr Metab (Lond). 2010; 7:55.

25. Singh RB, Rastogi SS, Ghosh S et al. The diet and moderate exercise trial (DAMET): results after 24 weeks. Acta Cardiol. 1992; 47:543-557. 
26. Nyantika AN, Tuomainen TP, Kauhanen J, Voutilainen S, Virtanen JK. Serum long-chain omega-3 polyunsaturated Fatty acids and future blood pressure in an ageing population. $\mathrm{J}$ Nutr Health Aging. 2015; 19(5):498-503.

27. Arbogast S, Ferreiro A. Selenoproteins and protection against oxidative stress: selenoprotein $\mathrm{N}$ as a novel player at the crossroads of redox signaling and calcium homeostasis. Antioxid Redox Signal. 2010;12(7):893-904.

28. Miller S, Walker SW, Arthur JR, Nicol F, Pickard K, Lewin MH, Howie AF, Beckett GJ. Selenite protects human endothelial cells from oxidative damage and induces thioredoxin reductase. Clin Sci (Lond). 2001; 100: 543-550.

29. Lymbury RS, Marino MJ, Perkins AV. Effect of dietary selenium on the progression of heart failure in the ageing spontaneously hypertensive rat. Mol Nutr Food Res. 2010; 54: 14361444.

30. Galan-Chilet I, Tellez-Plaza M, Guallar E, De Marco G, Lopez-Izquierdo R, GonzalezManzano I, et al. Plasma selenium levels and oxidative stress biomarkers: A geneenvironment interaction population-based study. Free Radic Biol Med. 2014; 74:229-236.

31. Ji A, Guo Y, Feng Z, Yang Q, Wang D, Yang W. Measurement of selenium content of nail and hair and correlation studies for Kaschin-Beck patients. Chinese Journal of Control of Endemic Disease. 1991; 6(6):327-328.

32. Ovaskainen ML, Virtamo J, Alfthan G, Haukka J, Pietinen P, Taylor PR, et al. Toenail selenium as an indicator of selenium intake among middle-aged men in an area with low soil selenium. Am J Clin Nutr. 1993; 57(5):662-665. 
33. Park K, Rimm EB, Siscovick DS, Spiegelman D, Manson JE, Morris JS, et al. Toenail selenium and incidence of type 2 diabetes in U.S. men and women. Diabetes Care. 2012; 35(7):1544-1951.

34. Vinceti M, Crespi CM, Malagoli C, Bottecchi I, Ferrari A, Sieri S, et al. A case-control study of the risk of cutaneous melanoma associated with three selenium exposure indicators. Tumori. 2012; 98(3):287-295. 
Table 1: Baseline characteristics of participants by nail selenium quintile groups.

\begin{tabular}{|c|c|c|c|c|c|c|c|}
\hline \multirow[b]{2}{*}{ Characteristics } & \multirow[b]{2}{*}{$\begin{array}{c}\text { Total } \\
(n=2000)\end{array}$} & \multicolumn{5}{|c|}{ Quintile groups of selenium level in nail samples $(\mu \mathrm{g} / \mathrm{g})$} & \multirow[b]{2}{*}{ P Value } \\
\hline & & $\begin{array}{c}\mathrm{Q} 1(\mathrm{n}=402) \\
(\leq 0.233)\end{array}$ & $\begin{array}{c}\text { Q2 }(n=405) \\
(0.234-0.362)\end{array}$ & $\begin{array}{c}\text { Q3 }(n=395) \\
(0.363-0.442)\end{array}$ & $\begin{array}{c}\mathrm{Q} 4(\mathrm{n}=397) \\
(0.443-0.552)\end{array}$ & $\begin{array}{c}\mathrm{Q} 5(\mathrm{n}=401) \\
(>0.552)\end{array}$ & \\
\hline $\begin{array}{l}\text { Nail selenium, } \\
(\mu \mathrm{g} / \mathrm{g})\end{array}$ & $\begin{array}{c}0.413 \pm 0.18 \\
3\end{array}$ & $0.187 \pm 0.030$ & $0.302 \pm 0.038$ & $0.407 \pm 0.022$ & $0.492 \pm 0.032$ & $0.683 \pm 0.143$ & $<0.0001$ \\
\hline Age (years) & $71.9 \pm 5.6$ & $72.0 \pm 5.6$ & $72.2 \pm 5.6$ & $71.9 \pm 5.5$ & $71.8 \pm 5.4$ & $71.6 \pm 5.7$ & 0.6858 \\
\hline Female $(\%)$ & 53.7 & 52.0 & 45.2 & 47.1 & 59.2 & 64.8 & $<0.0001$ \\
\hline $\begin{array}{l}\text { Attended school ( } \\
\%)\end{array}$ & 37.7 & 29.9 & 40.5 & 41.3 & 37.5 & 39.2 & 0.0061 \\
\hline $\begin{array}{l}\text { Alcohol consumer } \\
(\%)\end{array}$ & 43.6 & 59.7 & 50.9 & 41.5 & 32.2 & 33.2 & $<0.0001$ \\
\hline Smoker $(\%)$ & 46.4 & 45.0 & 51.1 & 53.2 & 41.6 & 41.2 & 0.0007 \\
\hline BMI $\left(\mathrm{kg} / \mathrm{m}^{2}\right)$ & $21.94 \pm 3.51$ & $21.05 \pm 2.70$ & $21.07 \pm 2.99$ & $21.78 \pm 3.56$ & $22.61 \pm 4.08$ & $23.19 \pm 3.55$ & $<0.0001$ \\
\hline $\begin{array}{l}\text { Systolic BP (mm } \\
\mathrm{Hg})\end{array}$ & $145.7 \pm 25.0$ & $133.8 \pm 20.8$ & $144.4 \pm 23.0$ & $148.6 \pm 25.8$ & $150.1 \pm 26.6$ & $151.6 \pm 24.4$ & $<0.0001$ \\
\hline $\begin{array}{l}\text { Diastolic BP (mm } \\
\mathrm{Hg})\end{array}$ & $83.6 \pm 12.7$ & $80.0 \pm 12.2$ & $84.1 \pm 12.3$ & $84.6 \pm 13.4$ & $84.5 \pm 13.5$ & $84.8 \pm 11.6$ & $<0.0001$ \\
\hline Hypertension (\%) & 63.50 & 43.78 & 62.22 & 66.33 & 69.52 & 75.81 & $<0.0001$ \\
\hline
\end{tabular}


Table2: Results from analysis of covariance (ANCOVA) model and logistic regression model on the association between nail selenium level, blood pressure measures and hypertension rate at baseline.

\begin{tabular}{|c|c|c|c|c|c|c|}
\hline \multirow{3}{*}{ Outcome variable } & \multicolumn{5}{|c|}{ Quintile groups of selenium level in nail samples $(\mu \mathrm{g} / \mathrm{g})$} & \multirow{3}{*}{ P Value } \\
\hline & Q1(n=402) & Q2 $(n=405)$ & Q3 $(n=395)$ & $\mathrm{Q} 4(\mathrm{n}=397)$ & $\mathrm{Q} 5(\mathrm{n}=401)$ & \\
\hline & $(\leq 0.233)$ & $(0.234-0.362)$ & $(0.363-0.442)$ & $(0.443-0.552)$ & $(>0.552)$ & \\
\hline \multicolumn{7}{|c|}{ Parameter Estimates from ANCOVA Models* } \\
\hline Systolic BP, mm Hg* & 0.0 (reference) & $10.87(7.58,14.16)$ & $14.45(11.09,17.81)$ & $15.26(11.86,18.66)$ & $16.05(12.65,19.45)$ & $<0.0001$ \\
\hline Diastolic BP, mm Hg* & 0.0 (reference) & $3.91(2.17,5.64)$ & $3.88(2.10,5.65)$ & $3.48(1.68,5.27)$ & $3.66(1.87,5.46)$ & $<0.0001$ \\
\hline \multicolumn{7}{|c|}{ Odds Ratios from Logistic Regression Model ${ }^{* *}$} \\
\hline Hypertension/Normal $* *$ & $1.0($ reference $)$ & $2.17(1.63,2.90)$ & $2.46(1.83,3.23)$ & $2.72(2.00,3.70)$ & $3.55(2.59,4.87)$ & $<0.0001$ \\
\hline
\end{tabular}

Note: * ANCOVA model adjusting for age, gender, BMI, education, smoking, and alcohol consumption.

**Logistic regression model adjusting for age, gender, BMI, education, smoking, and alcohol consumption. 
Table 3: Comparison of baseline characteristics between participants with incident hypertension and those normotensive during follow-up.

\begin{tabular}{lcccc}
\hline & $\begin{array}{c}\text { Overall } \\
(\mathrm{n}=635)\end{array}$ & $\begin{array}{c}\text { Normotensive } \\
(\mathrm{n}=275)\end{array}$ & $\begin{array}{c}\text { Incident Hypertension } \\
(\mathrm{n}=360)\end{array}$ & P Value \\
\hline Nail selenium, $(\mu \mathrm{g} / \mathrm{g})$ & $0.367 \pm 0.177$ & $0.340 \pm 0.188$ & $0.387 \pm 0.165$ & 0.0008 \\
Age (years) & $71.1 \pm 5.2$ & $71.1 \pm 5.3$ & $71.1 \pm 5.2$ & 0.8862 \\
Female (\%) & 51.97 & 52.36 & 51.67 & 0.8617 \\
Attended school (\%) & 35.43 & 38.18 & 33.33 & 0.2056 \\
Alcohol consumer (\%) & 44.41 & 47.27 & 42.22 & 0.2044 \\
Smoker (\%) & 48.03 & 48.36 & 47.78 & 0.8836 \\
BMI (kg/m $\left.{ }^{2}\right)$ & $21.05 \pm 3.07$ & $20.62 \pm 2.71$ & $21.39 \pm 3.28$ & 0.0017 \\
Systolic BP (mm Hg) & $123.3 \pm 10.3$ & $121.9 \pm 10.3$ & $124.4 \pm 10.3$ & 0.0018 \\
Diastolic BP (mm Hg) & $74.9 \pm 7.8$ & $74.6 \pm 7.3$ & $75.2 \pm 8.2$ & 0.3209 \\
\hline
\end{tabular}


Table 4: Hypertension incidence rate and hazard ratios for incident hypertension by selenium quintile groups during follow-up.

\begin{tabular}{lccc}
\hline Selenium quintile groups & Incidence rate $(\%)$ & Hazard ratio(95\% CI) & P- value \\
\hline Q1 (n=192) & 45.83 & 1.0 (reference) & -- \\
Q2 (n=132) & 52.27 & $1.41(1.03,1.94)$ & 0.0331 \\
Q3 (n=120) & 62.50 & $1.93(1.40,2.67)$ & $<0.0001$ \\
Q4 (n=105) & 70.48 & $2.35(1.69,3.26)$ & $<0.0001$ \\
Q5 (n=86) & 62.79 & $1.94(1.36,22.77)$ & 0.0002 \\
\hline
\end{tabular}

Note: Cox proportional hazards regression model adjusting for age, gender, BMI, education, smoking, alcohol consumption, and physical activity. 


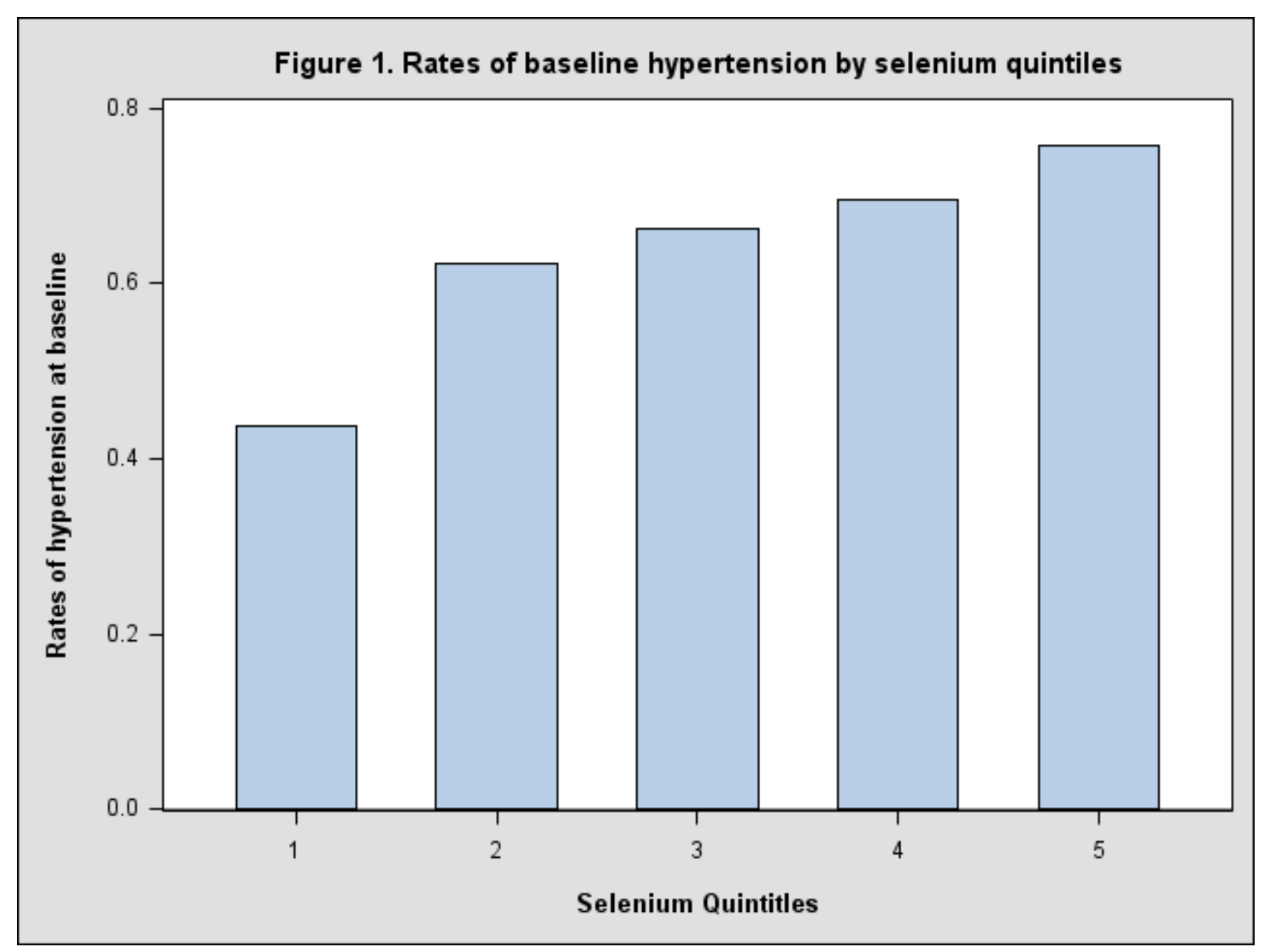




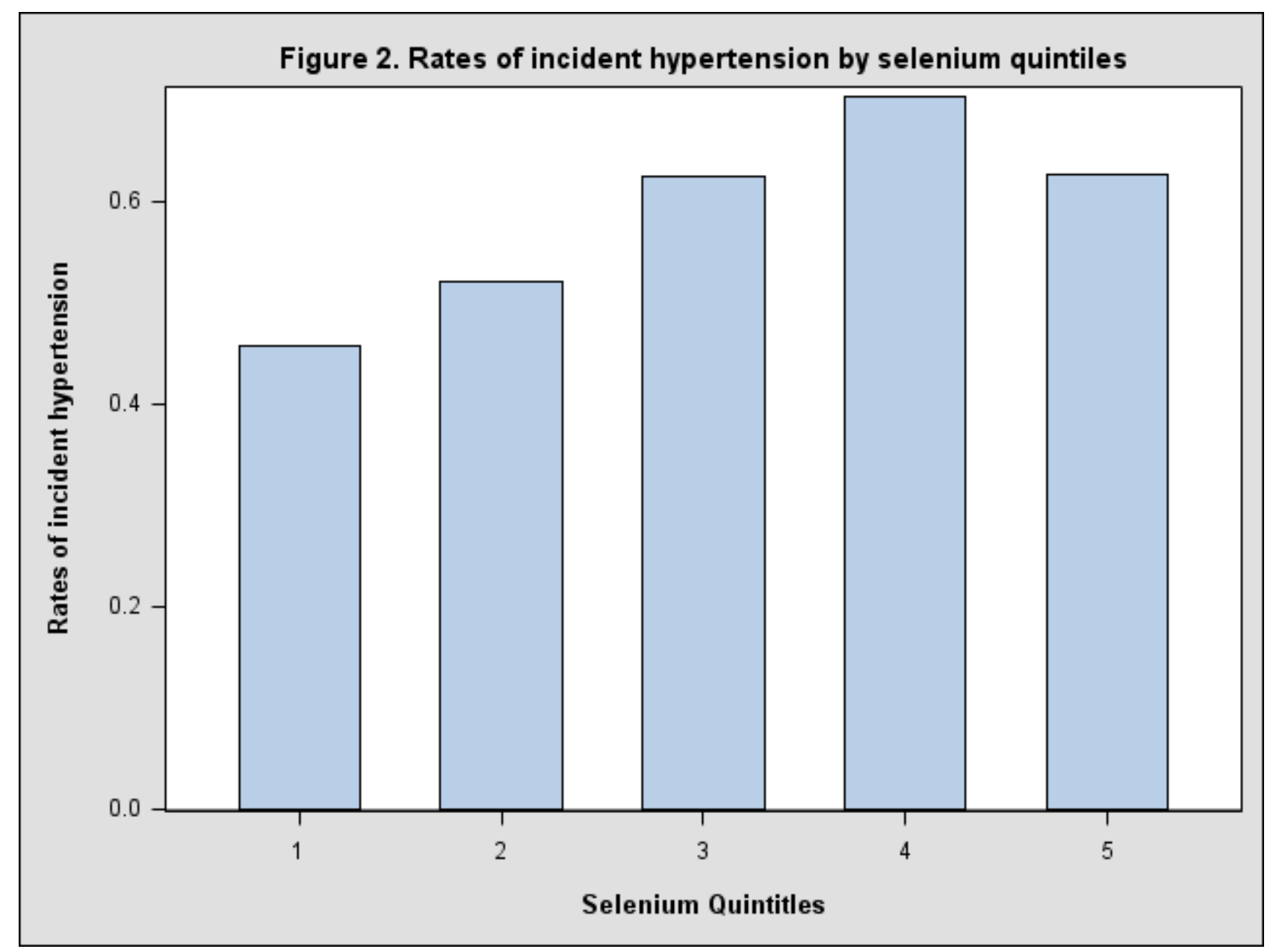

